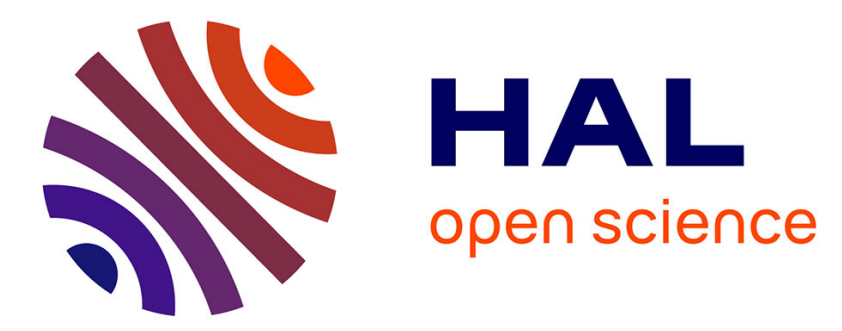

\title{
Ontology-Based Information Gathering System for Patients with Chronic Diseases: Lifestyle Questionnaire Design
}

Lamine Benmimoune, Amir Hajjam, Parisa Ghodous, Emmanuel Andres, Samy Talha, Mohamed Hajjam

\section{To cite this version:}

Lamine Benmimoune, Amir Hajjam, Parisa Ghodous, Emmanuel Andres, Samy Talha, et al.. Ontology-Based Information Gathering System for Patients with Chronic Diseases: Lifestyle Questionnaire Design. Progress in Artificial Intelligence, Sep 2015, Coimbra, Portugal. pp.110-115, 10.1007/978-3-319-23485-4_11. hal-01263333

\section{HAL Id: hal-01263333 \\ https://hal.science/hal-01263333}

Submitted on 27 Jan 2016

HAL is a multi-disciplinary open access archive for the deposit and dissemination of scientific research documents, whether they are published or not. The documents may come from teaching and research institutions in France or abroad, or from public or private research centers.
L'archive ouverte pluridisciplinaire HAL, est destinée au dépôt et à la diffusion de documents scientifiques de niveau recherche, publiés ou non, émanant des établissements d'enseignement et de recherche français ou étrangers, des laboratoires publics ou privés. 


\title{
Ontology-Based Information Gathering System for Patients with Chronic Diseases: Lifestyle Questionnaire Design
}

\author{
Lamine Benmimoune ${ }^{1,3}$, Amir Hajjam ${ }^{1}$, Parisa Ghodous ${ }^{2}$, Emmanuel Andres ${ }^{4}$, \\ Samy Talha ${ }^{4}$, and Mohamed Hajjam ${ }^{3}$ \\ 1 IRTES-SET, Université de Technologie Belfort-Montbéliard, 90000 Belfort, France \\ 2 LIRIS, Université Claude Bernard Lyon 1, 69100 Villeurbanne, France \\ 3 Newel, 68100 Mulhouse, France \\ 4 Hôpital civil de Strasbourg, 67000 Strasbourg, France \\ lamine.benmimoune@utbm.fr
}

\begin{abstract}
The aim of this paper is to describe an original approach which consists of designing an Information Gathering System (IGS). This system gathers the most relevant information related to the patient. Our IGS is based on using questionnaire ontology and adaptive engine which collects relevant information by prompting the whole significant questions in connection with the patient's medical background. The formerly collected answers are also taken into consideration in the questions selection process. Our approach improves the classical approach by customizing the interview to each patient. This ensures the selection of all of the most relevant questions. The proposed IGS is integrated within E-care monitoring platform for gathering lifestyle-related patient data.
\end{abstract}

Keywords: Information Gathering System, Questionnaire, Health-care, Clinical Decision Support System, Ontology, Monitoring

\section{Introduction}

Computer-based questionnaires are a new form of data collection, which are designed to offer more advantages compared to pen and paper questionnaires or oral interviewing [13. They are less time-consuming and more efficient by offering more structure and more details compared to the classical methods 2 .

The Information Gathering Systems (IGSs) have had measurable benefits in reducing omissions and errors arising as a result of medical interviews [14. The medical and health care domain is one of the most active domain in using IGS for gathering patients data [13].

Recently, various research works were conducted to design and to use the IGS as part of clinical decision support system (CDSS). Among them. Bouamrane et al. 2 2, 3] proposed a generic model for context-sensitive self-adaptation of IGS based on questionnaire ontology. The proposed model is implemented as a data collector module in 4 to collect patient medical history for preoperative 
risk assessment. Sherimon et al. [5, [6], [15] proposed an questionnaire ontology based on [2]. This ontology is used to gather patient medical history, which is then integrated within CDSS to predict the Risk of hypertension. Farooq et al. 7] proposed an ontology-based CDSS for chest pain risk assessment, based on [2] the proposed CDSS integrates a data collector to collect patient medical history. Alipour [13] proposed an approach to design an IGS based on the use of ontology-driven generic questionnaire and Pellet inference engine for questions selection process.

Although the presented IGS in the literature permit gathering patient data using ontologies, the created questionnaires are hard coded for specific domains and they are defined under the domain ontologies. These, make them less flexible, more difficult to maintain and even hard to share and to reuse.

Unlike previous approaches, our approach offers more flexibility by separating the ontologies and by integrating a domain ontology to drive the creation of questionnaire. This allows to give meaning to the created questions, and configuring different models of questionnaires without coding and regardless of the content of the domains. Therefore, many CDDSSs can easily integrate and use the proposed IGS for their specific needs.

Furthermore, the proposed approach permits to collect relevant information by prompting the whole significant questions in connection with the patient profile. The formerly collected answers are also taken into consideration in the questions selection process. This improves the classical approach by customizing the interview to each patient.

The proposed IGS is integrated within E-care home health monitoring platform [1], 8] for gathering lifestyle-related patient data.

\section{Information Gathering System within E-care platform}

E-care is a home health monitoring platform for patients with chronic diseases such as diabetes, heart failure, high blood pressure, etc. 1] 8. The aim is early detection of any anomalies or dangerous situations by collecting relevant data from the patient such as physiological data (heart rate, blood pressure, pulse, temperature, weight, etc.) and lifestyle data (tobacco-use, eating habits, physical activity, sleep, stress, etc.).

To improve the accuracy in anomalies detection, the platform needs relevant information that describes as precisely as possible the patient's health status and his lifestyle changes (tobacco-use, lack of physical activity, poor eating habits, etc.). That is why the patient is invited daily to collect his physiological data using medical sensors (Blood Pressure Monitor, Weighing Scale, Pulse Oximeter, etc.) and to answer on lifestyle questionnaires. These questionnaires are automatically generated by the IGS which permits gathering relevant information about the patient lifestyle.

All collected data (physiological data and lifestyle data) is stored in the patient profile ontology which models the health status of patient and then analysed by the inference engine for anomalies detection. 


\section{Information Gathering System Architecture}

The proposed architecture consists of four main components: Questionnaire Ontology, Survey History Ontology, Adaptive Engine and User Interfaces.

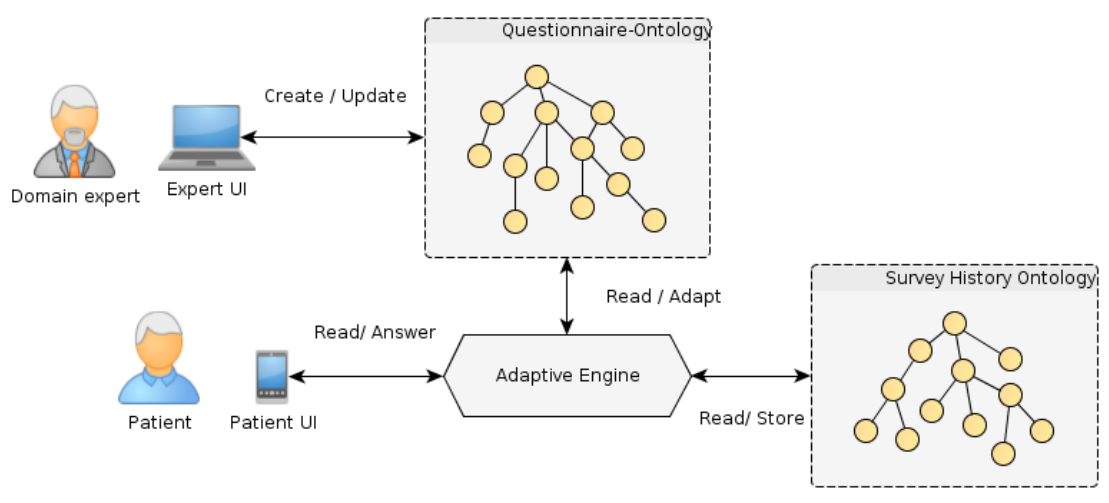

Fig. 1. Information Gathering System architecture

Questionnaire Ontology (QO): models the concepts representing the common components of a questionnaire. The QO is created based on Bouamrane et al.'s research works [2]. It is designed as generic, structured and flexible to accept most of the questionnaire models. The main classes are: Questionnaire, SubQuestionnaire, Question and PotentialAnswer. The Questionnaire class is composed of Sub-questionnaires, which represent a group of thematically related question classes. The question classes could be inter-related by structural properties such as hasParent, hasChild, hasSibling, etc. Each question is characterised by a type and related to one or more potential answers using Adaptive properties such as ifAnswerToThisQuestionEqualsTo, thenGoToQuestion, etc.

Survey History Ontology (SHO): stores all the patient surveys. It includes all the asked questions and the given answers by the patient. It is used in the questions selection process.

The Adaptive Engine (AE): it interprets the properties asserted in the questionnaire ontology and prompts the corresponding questions in connection with the patient profile and the formerly collected answers. The AE initially loads all questions except the children questions. It prompts the first question and checks if the question is appropriate to the patient profile (e.g. AE doesn't ask questions about the smoking habits, if the patient is a non-smoker). If it is, the AE asks 
the question and gets the answer from the UI. If it is not, the AE just prompts the next question.

If however the current question happens to be adaptive (i.e. it has at least a child question), the given answer is then checked against the answers that are expected to lead to children questions. If a match is found, the AE loads the children questions. If no match is found, the next question is prompted. The interaction loop is repeated until there are no more questions to be asked.

The User Interfaces (UI): consist of two parts of UI namely: expert UI and Patient UI.

- Expert UI: permits the domain experts (clinicians) to configure the IGS by defining questionnaires and to consult the surveys history.

- Patient UI: permits to start/stop the survey. It is designed in such a way that the patient can respond to the questionnaire from anywhere using his mobile device (tablet or smart phone).

\section{Domain Ontology Driven Questionnaire}

The domain ontology aims to drive the creation of questionnaires by offering a common and controlled vocabulary. To achieve this goal, we have developed a domain ontology for lifestyle concepts based on recommendations provided by Haute Autorité de Santé (HAS) 1 . The ontology is structured as an hierarchy of concepts and relations between concepts. It is composed of three main entities:

- LifeStyleEntities: hierarchical concepts that model lifestyle entities such as eating habits, physical activity, smoking habits, etc.

- DimensionsEntities: hierarchical concepts that model temporal dimensions and physical dimensions (quantity). Each dimension includes a hierarchy of concepts (e.g. TimesOfDay, TimeFrequency and TimeUnit are grouped under the timeDimension concept).

- CataloguesEntities: includes concepts used to give more semantic for the LifeStyleEntities concepts. Each catalogue entity includes a hierarchy of concepts that model the types of LifeStyleEntities concepts (e.g. Cigarette, Ecigarette and Drug are types of Tobacco concept for the SmokingHabits concept).

The concepts are related amongst them through properties as follows.

- DimensionProperties: used to relate the LifeStyleEntities to the DimensionsEntities, they include a set of properties such as hasQuantity, hasFrequency, hasTimesOfDay, etc.

- CatalogueProperties: used to relate the LifeStyleEntities to the CataloguesEntities, they include a set of properties such as hasExercise, hasTobacco, hasFood, etc.

\footnotetext{
${ }^{1}$ http:www.has-sante.fr
} 
The example illustrated by figure 2 shows how the domain concepts can be related amongst them and how they are used to design lifestyle questionnaires.

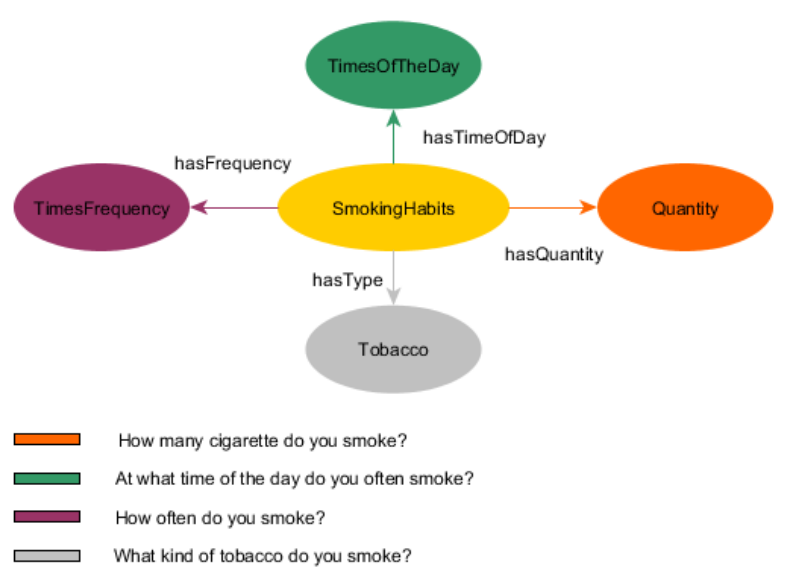

Fig. 2. Related questions for the "smoking habits" concept

Given the smoking habit that is characterized by a type of tobacco (e.g. cigarette, electronic cigarette, drugs, etc.), time frequency (daily, monthly, weekly, etc.), smoking quantity, etc. Several questions can be created based on SmokingHabits concept, with each smoking-related question should be related to the SmokingHabits through the domain properties, while the potential answers are related either to the DimensionsEntities or to the CatalogueProperties. (see figure 2).

\section{Conclusion and Future Work}

In this paper, we presented a novel approach, which aims to design an OntologyBased Information Gathering System. This system permits gathering the most relevant information by providing personalised questionnaires related to the patient profile. Our IGS consists of a questionnaire ontology which is driven by domain ontology. We have seen how the domain ontology is used to control vocabulary and to give a meaning to the asked questions. Furthermore, the use of a domain ontology can improve the gathering of data and the design of questionnaires can be made easier and faster compared to the hard coding of questionnaires. On the other hand, we have highlighted the interest of using the proposed IGS within E-care health monitoring platform, since it permits gathering relevant information about patients' lifestyle.

In the near future, we will experiment the proposed IGS in the real life with chronic patients. 


\section{References}

1. Benyahia, A.A., Hajjam, A., Hilaire, V., Hajjam, M.: E-care Ontological architecture for telemonitoring and alerts detection. 5 th IEEE International Symposium on Monitoring \& Surveillance Research (ISMSR): Healthcare-Safety-Security, (2012)

2. Bouamrane, M.M., Rector, A., Hurrell, M.: Ontology-Driven Adaptive Medical Information Collection System. 17th International Symposium, ISMIS 2008 Toronto, Canada, May 20-23, (2008)

3. Bouamrane, M.M., Rector, A., Hurrell, M.: Gathering Precise Patient Medical History with an Ontology-driven Adaptive Questionnaire. Computer-Based Medical Systems, 2008. CBMS '08. 21st IEEE International Symposium on 17-19 June (2008)

4. Bouamrane, M.M., Rector, A., Hurrell, M.: Using Ontologies for an Intelligent Patient Modelling, Adaptation and Management System. OTM 2008 Confederated International Conferences, CoopIS, DOA, GADA, IS, and ODBASE 2008, Monterrey, Mexico, November 9-14, (2008)

5. Sherimon, P.C., Vinu, P.V., Krishnan, R., Takroni, Y.: Ontology Based System Architecture to Predict the Risk of Hypertension in Related Diseases.IJIPM: International Journal of Information Processing and Management, Vol. 4, No. 4, pp. 44 50, (2013)

6. Sherimon, P.C., Vinu, P.V., Krishnan, R., Takroni,Y., AlKaabi,Y., AlFars, Y. :Adaptive Questionnaire Ontology in Gathering Patient Medical History in Diabetes Domain. Proceedings of the First International Conference on Advanced Data and Information Engineering (DaEng-2013) Lecture Notes in Electrical Engineering Volume 285, (2014)

7. Farooq, K, Hussain, A., Leslie, S., Eckl, C., Slack, W.: Ontology Driven Cardiovascular Decision Support System. Pervasive Computing Technologies for Healthcare (PervasiveHealth), 2011 5th International Conference on Date 23-26 May (2011)

8. Benyahia, A.A., Hajjam, A., Hilaire, V., Hajjam, M. , Andres, E.: E-care telemonitoring system: Extend the platform. Information, Intelligence, Systems and Applications (IISA), 2013 Fourth International Conference on 10-12 July (2013)

9. Saripalle, R.K.: Current status of ontologies in Biomedical and clinical Informatics., from University of Connecticut: http://www.engr.uconn.edu/ steve/Cse300/ saripalle.pdf Retrieved January 16,(2014)

10. Gruber, T.R.: Toward principles for the design of ontologies used for knowledge sharing., International Journal Human- Computer Studies Vol. 43, Issues 5-6, November 1995, Pages: 907-928. (1995)

11. Guarino, N.: Formal Ontology and Information Systems. Formal Ontology n Information Systems, Proceedings of FOIS98, Trento, Italy, 6-8 June (1998)

12. Noy, N.F., McGuinness, D.L.: Ontology Development 101: A Guide to Creating Your First Ontology. Stanford University, (2005)

13. Alipour-Aghdam, M.: Ontology-Driven Generic Questionnaire Design. Thesis for the degree of Master of Science in Computer Science. presented to The University of Guelph August, (2014)

14. BACHMAN, J.W.: The patient-computer interview: a neglected tool that can aid the clinician. Mayo Clinic Proceedings 78, 6778 (2003)

15. Sherimon, P.C, Vinu P.V, Krishnan, R., Saad, Y: Ontology driven analysis and prediction of patient risk in diabetes. Canadian Journal of Pure and Applied Sciences. SENRA Academic Publishers, British Columbia Vol. 8, No. 3, pp. 3043-3050, October (2014) 